\title{
Array Optimization for an On-The-Move 3D Imaging System Demonstrator
}

\author{
Borja Gonzalez-Valdes, Jose Vázquez-Cabo \\ Yolanda Rodriguez-Vaqueiro \\ AtlantTIC, Universidad de Vigo \\ Vigo, Spain
}

\author{
Yuri Álvarez, \\ Maria Garcia-Fernandez \\ University of Oviedo \\ Gijón Spain.
}

\author{
Ana Arboleya \\ University Rey Juan Carlos \\ Madrid Spain.
}

\begin{abstract}
In this work, array optimization for an imaging system demonstrator is presented. It is able to create 3D images of people as they walk through the scanner and it makes use of a very low number of transceivers. The array positions have been optimized to minimize grating lobes and maximize resolution for a given number of array elements.
\end{abstract}

\section{INTRODUCTION}

Next generation of security checkpoints will allow people under inspection to flow through them without interruption unless a potential threat is identified [1]. For this kind of application, active nearfield millimeter-wave (mm-wave) imaging radar systems are good candidates due to the fact that they are able to provide good resolution imaging with a reasonable cost [2]. Current systems [2] based on portal configuration are not particularly suitable for imaging people as they walk through the scanner. In [3], a multistatic radar architecture to provide On-The-Move imaging with a limited number of transmitters and receivers has been presented. A three-dimensional imaging system demonstrator to prove feasibility of this approach is currently under development. Preliminary two dimensional results were presented in [4]. In this work, the optimal size of the transmitting and receiving arrays for a given number of elements is obtained using Simulated Annealing optimization. The performance of the demonstrator is assessed using three dimensional Physical Optics-based simulations. Measurement results will be presented at the Conference.

\section{ARRAY SIZE OPTIMIZATION}

The layout of the demonstrator is depicted in Fig 1. It is composed by two arrays of transmitters (red dots) and receivers (blue circles) working on a fully coherent architecture and placed at $\mathbf{t}_{m}$ and $\mathbf{r}_{n}$ respectively.

The Object Under Test (OUT) is imaged as it moves through the hallway formed by the two opossite arrays [3]. Although this setup intrinsically minimizes the grating lobes due to the array undersampling [4], further reduction can be obtained by carefully selecting the transmitting and receiving array sizes and element positions [5]. To simplify the demonstrator manufacture only the arrays sizes are optimized, while the elements are equally spaced in a square array aperture. Of course, the larger the aperture, the larger the obtained crossrange resolution and area that can be imaged, but also the greater the array grating lobes when using sparse arrays. This

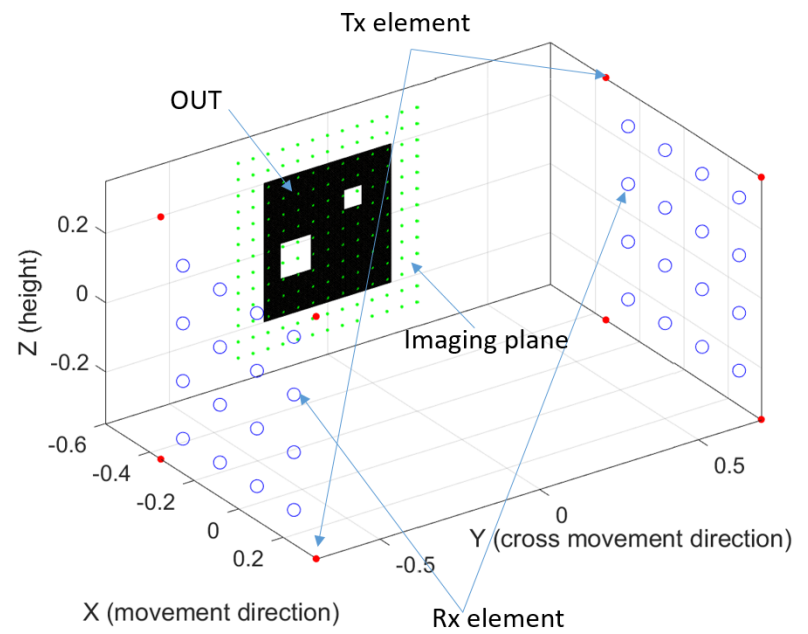

Fig. 1. Layout of the imaging system demonstrator.

trade-off can be expressed as a cost function depending on the Point Spread Function (PSF) of the system.

The total PSF of the system when focused in the point $\mathbf{p}_{i}^{u}$ can be calculated from the PSFs of the receiving and transmitting arrays, (1) and (2) respectively:

$$
\begin{aligned}
& P S F_{r x}\left(\mathbf{p}_{i}, \mathbf{p}_{i}^{u}\right)=\sum_{l, n} e^{j \kappa^{l}\left|\mathbf{p}_{n}-\mathbf{p}_{i}^{u}\right|} e^{-j \kappa^{l}\left|\mathbf{p}_{n}-\mathbf{p}_{i}\right|} \\
& P S F_{t x}\left(\mathbf{p}_{i}, \mathbf{p}_{i}^{u}\right)=\sum_{l, m} e^{j \kappa^{l}\left|\mathbf{t}_{m}-\mathbf{p}_{i}^{u}\right|} e^{-j \kappa^{l}\left|\mathbf{t}_{m}-\mathbf{p}_{i}\right|} \\
& P S F_{\text {total }}\left(\mathbf{p}_{i}, \mathbf{p}_{i}^{u}\right)=P S F_{r x}\left(\mathbf{p}_{i}\right) * P S F_{t x}\left(\mathbf{p}_{i}\right)
\end{aligned}
$$

where multiple frequencies $f_{l}$ are used to create the images in Ultrawide Band Radar (UWB) radar configuration, and $\kappa^{l}$ is the wavenumber at the $l$-th frequency. The imaging plane pixels are denoted as $\mathbf{p}_{i}$.

In the cost function, both the size of the PSF's main beam and the grating lobes are considered. The obtained cost function is maximized using a Simulated Annealing algorithm [6]. This PSF is calculated in different focusing points $\mathbf{p}_{i}^{u}$ on the imaging plane pixels depicted as green dots in Fig. 1] Since the system must be able to focus at different distances from 
the array, the PSF is tested at imaging planes placed at 60 , 70 and $80 \mathrm{~cm}$ from the array center. The different distances and focusing points are averaged to build the cost function for each set of transmitters/receivers positions:

$$
\begin{aligned}
\operatorname{Cost}\left(\mathbf{t}_{m}, \mathbf{r}_{n}\right)= & \sum_{1}^{N_{d}} \sum_{1}^{N u} C_{1} P S F_{\text {total }}^{d}\left(\mathbf{p}_{i}, \mathbf{p}_{i}^{u}\right)_{S L L}+ \\
& +\sum_{1}^{N_{d}} \sum_{1}^{N u} \frac{C_{2}}{P S F_{\text {total }}^{d}\left(\mathbf{p}_{i}, \mathbf{p}_{i}^{u}\right)_{B W}}
\end{aligned}
$$

where $P S F_{\text {total }}^{d}\left(\mathbf{p}_{i}, \mathbf{p}_{i}^{u}\right)_{S L L}$ and $P S F_{\text {total }}^{d}\left(\mathbf{p}_{i}, \mathbf{p}_{i}^{u}\right)_{B W}$ are respectively the maximum Side Lobe Level and $-3 \mathrm{~dB}$ beamwidth of the PSF at the $d$-th distance and focused on the $u$-th point on the imaging plane. $C_{1}$ and $C_{2}$ are constants used to weight in the two different parts of the cost function.

$8 \mathrm{Tx}$ and $32 \mathrm{Rx}$ channels are currently available to build the imaging system demonstrator. Thus this is the number of positions used in the arrays optimization. An UWB system with frequencies ranging from 5 to $18 \mathrm{GHz}$ is considered. The frequency sweep is obtained in the prototype using a Vector Network Analyzer (VNA) and this BW is mainly restricted by the characteristics of the Vivaldi antennas that are being used to build the prototype (Fig 2). Three wideband RF switches are used to sequentially multiplex the 40 antennas into the four VNA ports.

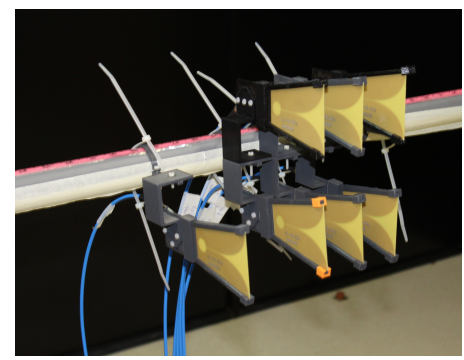

Fig. 2. Picture of the Vivaldi antennas used in the $2 \mathrm{D}$ array of the prototype.

\section{RESULTS}

Fig. 3 presents the optimized PSF according the procedure described above. The optimized arrays positions are presented in Fig. 1. Their size is $69 \mathrm{~cm}$ for the transmitting array and 55 $\mathrm{cm}$ for the receiving one, obtaining a $1.6 \mathrm{~cm}$ wide PSF at 70 $\mathrm{cm}$ from the array center with average SLL of $30 \mathrm{~dB}$. Fig. 4 shows the obtained radar image using backpropagation [5] for the OUT in Fig. 1, a 40x40 cm metal plate with two square holes (of 10 and $6 \mathrm{~cm}$ side) placed $60 \mathrm{~cm}$ from the arrays centers. Good imaging capabilities are demonstrated using a very limited number of transceivers.

\section{ACKNOWLEDGEMENT}

This work is partially supported by the Spanish National Research and Development Program project TEC2015-73908JIN and RYC-2016-20280, by the European Regional Development Fund (ERDF), and by the Galician Government under project GRC2015/018 and agreement for funding AtlantTIC.

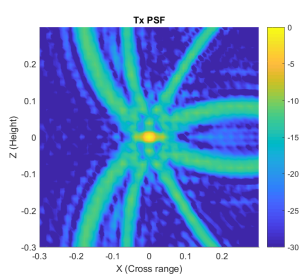

(a)

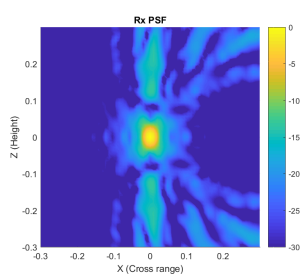

(b)

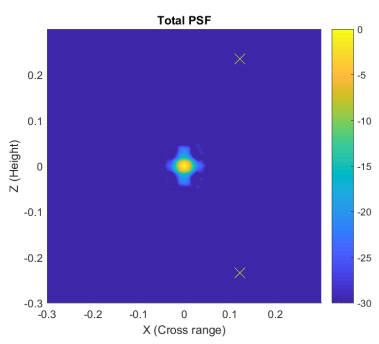

(c)

Fig. 3. Results of the optimization (a) transmitting array PSF (b) receiving array PSF (c) total PSF. Yellow crosses show the location of the highest side lobes

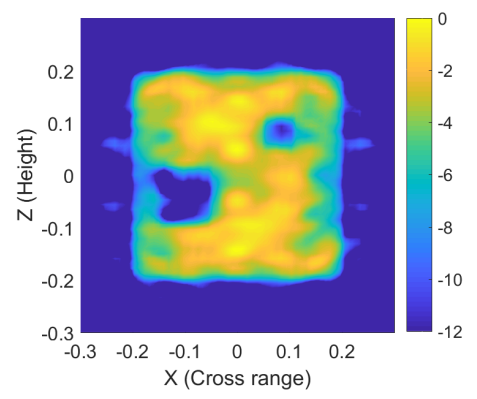

Fig. 4. Obtained radar image (reflectivity in $\mathrm{dB}$ ) of the simulated OUT.

\section{REFERENCES}

[1] IATA, "Checkpoint of the future. executive summary." Available at: http://www.iata.org/whatwedo/security/Documents/cof-executivesummary.pdf (Latest accessed on 03.16.15.).

[2] S. S. Ahmed, "Personnel screening with advanced multistatic imaging technology," in SPIE Defense, Security, and Sensing. International Society for Optics and Photonics, 2013, pp. 87 150B-87 150B.

[3] B. Gonzalez-Valdes, Y. Alvarez, Y. Rodriguez-Vaqueiro, A. ArboleyaArboleya, A. Pino, C. M. Rappaport, F. Las-Heras, and J. A. MartinezLorenzo, "Millimeter wave imaging architecture for on-the-move whole body imaging," IEEE Transactions on Antennas and Propagation, vol. 64, no. 6, pp. 2328-2338, June 2016.

[4] B. Gonzalez-Valdes, Y. Rodriguez-Vaqueiro, Y. Alvarez, F. L. Heras, and A. P. Garcia, "Nearfield-based array design for a realistic on-themove personnel inspection system," in 2017 11th European Conference on Antennas and Propagation (EUCAP), March 2017, pp. 2846-2850.

[5] X. Zhuge and A. Yarovoy, "A sparse aperture mimo-sar-based uwb imaging system for concealed weapon detection," Geoscience and Remote Sensing, IEEE Transactions on, vol. 49, no. 1, pp. 509-518, 2011.

[6] B. Gonzalez-Valdes, G. Allan, Y. Rodriguez-Vaqueiro, Y. lvarez, S. Mantzavinos, M. Nickerson, B. Berkowitz, J. A. Martnez-Lorenzo, F. Las-Heras, and C. M. Rappaport, "Sparse array optimization using simulated annealing and compressed sensing for near-field millimeter wave imaging," IEEE Transactions on Antennas and Propagation, vol. 62 , no. 4, pp. 1716-1722, April 2014 